\title{
Unitary toy qubit transport model for black hole evaporation
}

\author{
Bogusław Broda ${ }^{\mathrm{a}}$ \\ Department of Theoretical Physics, Faculty of Physics and Applied Informatics, University of Łódź, Pomorska 149/153, 90-236 Lodz, Poland
}

Received: 5 December 2019 / Accepted: 19 April 2020 / Published online: 13 May 2020

(C) The Author(s) 2020

\begin{abstract}
In a recent paper Osuga and Page have presented an explicitly unitary toy qubit transport model for transferring information from a black hole to the outgoing radiation. Following their idea we propose a unitary toy model which involves (fermionic) Hawking states.
\end{abstract}

\section{Introduction}

The black hole information (loss) paradox/problem/puzzle concerns difficulties in answering the question: "Is black hole evaporation unitary?" Various answers and explanations have been proposed to date, "for" its unitarity as well as "against" it (for recent reviews see [1-4]). (The latter possibility, i.e., loss of unitarity, presumably requires some radical modifications of quantum mechanics, and presently this option seems to be less popular, however see [5]). To analyze the issue of unitarity in the context of black hole evaporation, the idea of toy qubit models has been proposed, developed and popularized, especially in [6-10], and nicely reviewed in [11]. Toy qubit models give a possibility to mimic, in a simplified way, black hole evolution, and to trace, in detail, their (depending on the model) breaking or maintaining unitarity.

In a recent paper [12], Osuga and Page have proposed a strikingly simple toy qubit model demonstrating transferring information from a black hole to the outgoing radiation, which is explicitly unitary. Characteristic features of their model, in particular, include: ( $i$ ) Hilbert spaces (implicitly) involved in their analysis are actually fixed, though in their final state $\left|\Psi_{1}\right\rangle$ some part is dropped; (ii) the model has a simple (tensor) product structure (no mixing of different modes " $i$ "); and most noteworthy, (iii) unitarity of their model is shown in the most direct way, i.e., a corresponding unitary operator is explicitly constructed.

\footnotetext{
a e-mail: boguslaw.broda@uni.lodz.pl (corresponding author) URL: http://merlin.phys.uni.lodz.pl/BBroda
}

In the present paper, we propose another toy qubit transport model for black hole evaporation, which is also explicitly unitary. Additional nice characteristic features include: (i) precisely (fermionic) Hawking states are involved; (ii) classical shrinking of a black hole, in quantum formalism, corresponds to transition to a vacuum state (in the black hole sector); (iii) primary entanglement (in the Hawking states) between modes inside and outside of the black hole vanishes.

For reader's convenience, as well as for ours, we follow conventions and notation of [12] as closely as possible. In particular, we denote black hole qubits (once the black hole forms) by $a_{i}$, where $i=1,2, \ldots, n$, whereas qubits for the infalling radiation and for the outgoing modes, by $b_{i}$ and $c_{i}$, respectively, where $i=1,2, \ldots, N$. For technical simplicity, from now on we confine ourselves to a Schwarzschild black hole, but this restriction is not crucial for our further considerations.

\section{The model}

In the beginning, to each black hole mode $a_{i}$, we associate a (fermionic) Hawking state $\left|H\left(\Omega_{i}\right)\right\rangle_{b_{i} c_{i}}$, i.e., a pair of entangled radiation qubits, $b_{i}$ (infalling) and $c_{i}$ (outgoing), in the state

$\left|H\left(\Omega_{i}\right)\right\rangle_{b_{i} c_{i}}=\cos \Omega_{i}|0\rangle_{b_{i}}|0\rangle_{c_{i}}+\sin \Omega_{i}|1\rangle_{b_{i}}|1\rangle_{c_{i}}$

which is precisely the fermionic state created by a black hole, according to the Hawking effect. Alternatively, we could interpret (1) in terms of a two-term (qualitative) approximation of the bosonic Hawking state (see, e.g., the second sentence after Eq. (2.2) in [13]). The parameter $\Omega_{i}$ is a function of Bogoljubov coefficients following from spacetime geometry. From more physical point of view, $\Omega_{i}$ is a $i$-mode dependent function of gravitational field embodied (in the Schwarzschild case) by the black hole mass $M_{\text {bh }}$. The above association is equivalent to the assumption that 
the black hole modes $a_{i}$ and the Hawking states (1) can be paired, i.e., $N=n$. It is true (or even obvious), at least approximately, i.e. $N \sim n$. Indeed, the number of black hole modes $n \sim S_{\mathrm{BH}}=4 \pi M_{\mathrm{bh}}^{2}$, where $S_{\mathrm{BH}}$ is the Bekenstein-Hawking entropy (we have used Planck units in which $\hbar=c=G=k_{\text {Boltzmann }}=1$ ), whereas estimated number of outgoing radiation modes $N \sim M_{\mathrm{bh}}^{2}$ (see [14,15]). Consequently, the initial quantum state of black hole modes and Hawking radiation is (cf. [12])

$$
\left|\Psi_{0}\right\rangle=\sum_{q_{1}, q_{2}, \ldots, q_{n}=0}^{1} A_{q_{1} q_{2} \cdots q_{n}} \prod_{i=1}^{n}\left|q_{i}\right\rangle_{a_{i}} \prod_{i=1}^{n}\left|H\left(\Omega_{i}\right)\right\rangle_{b_{i} c_{i}},
$$

where $A_{q_{1} q_{2} \cdots q_{n}}$ are the amplitudes for the black hole modes $a_{i}$.

Let us note that the state $\left|\Psi_{0}\right\rangle$ is a tensor product of $n+1$ (block-)states, which are, in general, entangled states. Schematically, we can express it explicitly as $\left|\Psi_{0}\right\rangle=|A\rangle \otimes$ $|H\rangle_{1} \otimes \cdots \otimes|H\rangle_{n}$.

The elementary, i.e., for fixed $i$, process we propose to consider is (cf. Eq. (3.3) in [12], and possibly also Eq. (3.3) in [16])

$\left|q_{i}\right\rangle_{a_{i}}\left|H\left(\Omega_{i}\right)\right\rangle_{b_{i} c_{i}} \longmapsto|0\rangle_{a_{i}}|0\rangle_{b_{i}}\left|q_{i}\right\rangle_{c_{i}}$.

Obviously, the whole qubit transport process is a ("tensor") product of $n$ processes of the type (3) for each mode $i$. Evidently, the process (3) transports information (which could be previously scrambled by a unitary evolution of the black hole) encoded in the (base) qubits $\left|q_{i}\right\rangle\left(q_{i}=0,1\right)$ from the black hole modes $a_{i}$ to the outgoing radiation modes $c_{i}$. Moreover, the mode levels inside the black hole $\left(a_{i}\right.$ and $\left.b_{i}\right)$ become gradually emptied (classically, the black hole gradually shrinks), and furthermore, the primarily entangled initial state on the LHS of (3) becomes unentangled on the RHS.

\section{Unitarity}

To explicitly show unitarity of the qubit transformation (3), it is sufficient to construct a corresponding unitary operator performing the required transformation (3). To this end, let us first define two auxiliary (parameter-dependent) orthonormal bases in the tensor product Hilbert space $\mathcal{H}_{i}=\mathcal{H}_{a_{i}} \times \mathcal{H}_{b_{i}} \times$ $\mathcal{H}_{c_{i}}$, where we have introduced the three Hilbert spaces (of complex dimension 2, each) for all types of the involved modes: $a_{i} \in \mathcal{H}_{a_{i}}, b_{i} \in \mathcal{H}_{b_{i}}, c_{i} \in \mathcal{H}_{c_{i}}\left(\operatorname{dim}_{\mathbb{C}} \mathcal{H}_{i}=8\right)$. The total Hilbert space is then $\mathcal{H}=\bigotimes_{i=1}^{n} \mathcal{H}_{i}$.

The first (unprimed) orthonormal base parameterized by $\omega_{i}$ is $\left\{\left|E_{\Lambda}\left(\omega_{i}\right)\right\rangle_{a_{i} b_{i} c_{i}}\right\}_{\Lambda=0}^{7}$, and it consists of the following set of states/vectors:

$$
\begin{aligned}
\left|E_{0}\right\rangle_{a_{i} b_{i} c_{i}} & =|0\rangle_{a_{i}}\left(\cos \omega_{i}|0\rangle_{b_{i}}|0\rangle_{c_{i}}+\sin \omega_{i}|1\rangle_{b_{i}}|1\rangle_{c_{i}}\right) \\
\left|E_{1}\right\rangle_{a_{i} b_{i} c_{i}} & =|0\rangle_{a_{i}}|0\rangle_{b_{i}}|1\rangle_{c_{i}} \\
\left|E_{2}\right\rangle_{a_{i} b_{i} c_{i}} & =|0\rangle_{a_{i}}|1\rangle_{b_{i}}|0\rangle_{c_{i}} \\
\left|E_{3}\right\rangle_{a_{i} b_{i} c_{i}} & =|0\rangle_{a_{i}}\left(-\sin \omega_{i}|0\rangle_{b_{i}}|0\rangle_{c_{i}}+\cos \omega_{i}|1\rangle_{b_{i}}|1\rangle_{c_{i}}\right) \\
\left|E_{4}\right\rangle_{a_{i} b_{i} c_{i}} & =|1\rangle_{a_{i}}\left(\cos \omega_{i}|0\rangle_{b_{i}}|0\rangle_{c_{i}}+\sin \omega_{i}|1\rangle_{b_{i}}|1\rangle_{c_{i}}\right) \\
\left|E_{5}\right\rangle_{a_{i} b_{i} c_{i}} & =|1\rangle_{a_{i}}|0\rangle_{b}|1\rangle_{c_{i}} \\
\left|E_{6}\right\rangle_{a_{i} b_{i} c_{i}} & =|1\rangle_{a_{i}}|1\rangle_{b_{i}}|0\rangle_{c_{i}} \\
\left|E_{7}\right\rangle_{a_{i} b_{i} c_{i}} & =|1\rangle_{a_{i}}\left(-\sin \omega_{i}|0\rangle_{b_{i}}|0\rangle_{c_{i}}+\cos \omega_{i}|1\rangle_{b_{i}}|1\rangle_{c_{i}}\right) .
\end{aligned}
$$

The second (primed) orthonormal base parameterized by $\theta_{i}$ is $\left\{\left|E_{\Lambda}^{\prime}\left(\theta_{i}\right)\right\rangle_{a_{i} b_{i} c_{i}}\right\}_{\Lambda=0}^{7}$, and it is defined as:

$$
\begin{aligned}
\left|E_{0}^{\prime}\right\rangle_{a_{i} b_{i} c_{i}} & =|0\rangle_{a_{i}}|0\rangle_{b_{i}}|0\rangle_{c_{i}} \\
\left|E_{1}^{\prime}\right\rangle_{a_{i} b_{i} c_{i}} & =\cos \frac{\theta_{i}}{2}|0\rangle_{a_{i}}|0\rangle_{b_{i}}|1\rangle_{c_{i}}-\sin \frac{\theta_{i}}{2}|1\rangle_{a_{i}}|0\rangle_{b_{i}}|0\rangle_{c_{i}} \\
\left|E_{2}^{\prime}\right\rangle_{a_{i} b_{i} c_{i}} & =|0\rangle_{a_{i}}|1\rangle_{b_{i}}|0\rangle_{c_{i}} \\
\left|E_{3}^{\prime}\right\rangle_{a_{i} b_{i} c_{i}} & =|0\rangle_{a_{i}}|1\rangle_{b_{i}}|1\rangle_{c_{i}} \\
\left|E_{4}^{\prime}\right\rangle_{a_{i} b_{i} c_{i}} & =\sin \frac{\theta_{i}}{2}|0\rangle_{a_{i}}|0\rangle_{b_{i}}|1\rangle_{c_{i}}+\cos \frac{\theta_{i}}{2}|1\rangle_{a_{i}}|0\rangle_{b_{i}}|0\rangle_{c_{i}} \\
\left|E_{5}^{\prime}\right\rangle_{a_{i} b_{i} c_{i}} & =|1\rangle_{a_{i}}|0\rangle_{b}|1\rangle_{c_{i}} \\
\left|E_{6}^{\prime}\right\rangle_{a_{i} b_{i} c_{i}} & =|1\rangle_{a_{i}}|1\rangle_{b_{i}}|0\rangle_{c_{i}} \\
\left|E_{7}^{\prime}\right\rangle_{a_{i} b_{i} c_{i}} & =|1\rangle_{a_{i}}|1\rangle_{b_{i}}|1\rangle_{c_{i}} .
\end{aligned}
$$

The explicitly unitary transformation $U_{i}\left(\theta_{i}\right)$ can now be constructed from the two bases (4) and (5) in a standard way as

$$
U_{i}\left(\theta_{i}\right)=\sum_{\Lambda=0}^{7}\left|E_{\Lambda}^{\prime}\left(\theta_{i}\right)\right|_{a_{i} b_{i} c_{i}}\left\langle\left. E_{\Lambda}\left(\pi^{-1} \theta_{i} \Omega_{i}\right)\right|_{a_{i} b_{i} c_{i}},\right.
$$

where the auxiliary parameter ("time") $\theta_{i}$ satisfies: $0 \leq \theta_{i} \leq$ $\pi$ (cf. [12]). Evidently, for $\theta_{i}=\pi$, the unitary operator (6) performs the required transformation (3).

Thus, finally, expressing the total unitary transformation as a tensor product $U(\pi)=\bigotimes_{i=1}^{n} U_{i}(\pi)$, we can write $U(\pi)\left|\Psi_{0}\right\rangle=\left|\Psi_{1}\right\rangle$, where the final state $\left|\Psi_{1}\right\rangle$ assumes the explicit form

$$
\left|\Psi_{1}\right\rangle=\prod_{i=1}^{n}|0\rangle_{a_{i}} \prod_{i=1}^{n}|0\rangle_{b_{i}} \sum_{q_{1}, q_{2}, \ldots, q_{n}=0}^{1} A_{q_{1} q_{2} \cdots q_{n}} \prod_{i=1}^{n}\left|q_{i}\right\rangle_{c_{i}} .
$$

It follows from Eq. (7) that the whole black hole information encoded in the amplitudes $A_{q_{1} q_{2} \cdots q_{n}}$ has been transferred from the black hole modes $a_{i}$ to the outgoing radiation modes $c_{i}$, whereas the black hole modes $a_{i}, b_{i}$ are now in the vacuum state. Moreover, there is no entanglement between modes inside and outside of the black hole. 
One could wonder what happens if the final state $\left|\Psi_{1}\right\rangle$ is "time-evolved" further. First of all, we should emphasize that the proposed model is supposed to refer only to a prescribed period of time. In the assumed units, it is $0 \leq \theta \leq \pi$. Therefore, at the end of the time evolution all $a_{i}$ - and $b_{i}$-modes are (see (7)) in a vacuum state. But if we, purely formally, time-evolved the final state further using Eq. (6) outside the domain of its supposed applicability, due to the presence of trigonometric functions in (4) and (5), we would observe (mode-dependent) oscillatory character of the evolution. In particular, $a_{i}$ - and $b_{i}$-states could get switched on again.

\section{Final remarks}

The auxiliary parameter(s) $\theta_{i}$ can be used in (6) for two purposes. First of all, one can apply $\theta_{i}$ to gradually switchon the process (3); e.g., $\theta_{i}$ could be some decreasing functions of a curvature invariant as in [12]. Alternatively, one could possibly try to extract a corresponding "Hamiltonian" $\boldsymbol{H}$ (cf. Eq. (3.4) in [12]) from the "short-time" limit: $U(\theta)=I-i \theta \boldsymbol{H}$. However, the latter procedure is highly non-unique (see the following paragraph).

We would like to draw the reader's attention to a minor technical detail. Namely, the unitary operator (6) is introduced in a highly non-unique way, in the sense that there is a large group of ( $i$-dependent) unitary transformations (which could be implemented, e.g., in terms of unitary transformations on some subsets of vectors of the bases) changing the operator itself but still performing the same transformation (3) (obviously, the same is true for the operator proposed by Osuga and Page in [12]). This non-uniqueness follows from the fact that there is large freedom in determining the unitary operator - the freedom is only restricted by the condition/process (3) which involves only some very particular states. The action of the operator on other states is undetermined by (3), and hence it is arbitrary.

Recapitulating, as a final remark, we would like to add that our idea to use precisely the state (2) as an initial state (with fermionic Hawking states included), and (7) as a final state (with vacua for the black hole modes $a_{i}$ and $b_{i}$ ) follows from the fact that it was our intention to have a possibility to interpret qubits a little bit more realistically, as possible fermionic particles, rather than as only purely formal entities.

Data Availability Statement This manuscript has no associated data or the data will not be deposited. [Authors' comment: There is no data related to this publication that could be deposited. A preprint version of this article was published as at arXiv:1911.08295[hep-th].]

Open Access This article is licensed under a Creative Commons Attribution 4.0 International License, which permits use, sharing, adaptation, distribution and reproduction in any medium or format, as long as you give appropriate credit to the original author(s) and the source, provide a link to the Creative Commons licence, and indicate if changes were made. The images or other third party material in this article are included in the article's Creative Commons licence, unless indicated otherwise in a credit line to the material. If material is not included in the article's Creative Commons licence and your intended use is not permitted by statutory regulation or exceeds the permitted use, you will need to obtain permission directly from the copyright holder. To view a copy of this licence, visit http://creativecomm ons.org/licenses/by/4.0/.

Funded by $\mathrm{SCOAP}^{3}$.

\section{References}

1. Daniel Harlow, Jerusalem lectures on black holes and quantum information. Rev. Mod. Phys. 88, 015002 (2016). https://doi.org/ 10.1103/RevModPhys.88.015002

2. D. Marolf, The Black Hole information problem: past, present, and future. Rep. Prog. Phys. 80(9), 092001 (2017). https://doi.org/10. 1088/1361-6633/aa77cc

3. J. Polchinski. The black hole information problem. In: Proceedings, Theoretical Advanced Study Institute in Elementary Particle Physics: New Frontiers in Fields and Strings (TASI 2015): Boulder, CO, USA, June 1-26, 2015 (2017), pp. 353-397. https://doi.org/ $10.1142 / 10270$

4. S. Chakraborty, K. Lochan, Black holes: eliminating information or illuminating new physics? Universe 3(3), 55 (2017). https://doi. org/10.3390/universe3030055

5. W.G. Unruh, R.M. Wald, Information loss. Rep. Prog. Phys. 80(9), 092002 (2017). https://doi.org/10.1088/1361-6633/aa778e

6. S.B. Giddings, Black holes, quantum information, and unitary evolution. Phys. Rev. D 85, 124063 (2012a). https://doi.org/10.1103/ PhysRevD.85.124063

7. S.B. Giddings, Models for unitary black hole disintegration. Phys. Rev. D 85, 044038 (2012b). https://doi.org/10.1103/PhysRevD.85. 044038

8. S.B. Giddings, Y. Shi, Quantum information transfer and models for black hole mechanics. Phys. Rev. D 87(6), 064031 (2013). https://doi.org/10.1103/PhysRevD.87.064031

9. S.D. Mathur, The information paradox: a pedagogical introduction. Class. Quantum Gravity 26, 224001 (2009a). https://doi.org/10. 1088/0264-9381/26/22/224001

10. S.D. Mathur, What exactly is the information paradox? Lect. Notes Phys. 769, 3-48 (2009b). https://doi.org/10.1007/ 978-3-540-88460-6_1

11. S.G. Avery, Qubit models of black hole evaporation. JHEP 01, 176 (2013). https://doi.org/10.1007/JHEP01(2013)176

12. K. Osuga, D.N. Page, Qubit transport model for unitary black hole evaporation without firewalls. Phys. Rev. D 97(6), 066023 (2018). https://doi.org/10.1103/PhysRevD.97.066023

13. S.D. Mathur, C.J. Plumberg, Correlations in Hawking radiation and the infall problem. JHEP 09, 093 (2011). https://doi.org/10.1007/ JHEP09(2011)093

14. W. Mück, Hawking radiation is corpuscular. Eur. Phys. J. C 76(7), 374 (2016). https://doi.org/10.1140/epjc/s10052-016-4233-3

15. B. Broda, Total spectral distributions from Hawking radiation. Eur. Phys. J. C 77(11), 756 (2017). https://doi.org/10.1140/epjc/ s10052-017-5336-1

16. A. Almheiri, D. Marolf, J. Polchinski, D. Stanford, J. Sully, An apologia for firewalls. JHEP 09, 018 (2013). https://doi.org/10. 1007/JHEP09(2013)018 